Chirurgia (2021) 116: 51-59

No. 1, January - February

Copyright@ Celsius

http://dx.doi.org/10.21614/chirurgia.116.1.51

\title{
Is BMI a Factor in Compliance to Adjuvant Chemotherapy for Locally Advanced Rectal Cancer?
}

\author{
A multicenter retrospective analysis and comparison with non-obese patients
}

\begin{abstract}
Giulio M. Mari' ${ }^{1 *}$, Jacopo Crippa ${ }^{2}$, Achilli Pietro², Dario Maggioni', Andrea Costanzi', Mauro A. Scotti', Marco Braga', Eugenio Cocozza ${ }^{3}$, Giacomo Borroni ${ }^{3}$, Ilaria Benzoni', Luigi Totaro ${ }^{4}$, Matteo Origi', Giovanni Ferrari², Antonio Ziccarelli ${ }^{5}$, Roberto Petri ${ }^{5}$, Vincenzo Bagnardi ${ }^{6}$, on behalf of the AIMS Academy Clinical Research Network [corporate author]
\end{abstract}

1Department of General Surgery, ASST Monza, Desio Hospital, Italy

2Department of General Surgery, Niguarda Hospital, Milano, Italy

${ }^{3}$ Department of General Surgery, ASST Insubria, Varese Hospital, Italy

${ }^{4}$ Department of General Surgery, ASST Cremona, Cremona Hospital, Italy

${ }^{5}$ Department of General Surgery, ASUI Udine, University Hospital, Italy

${ }^{6}$ Statistical Departement, Bicocca Univeristy, Milano, Italy

${ }^{*}$ Corresponding author:

Mari Giulio MD

General Surgery Departement

ASST Monza, Desio Hospital

Via Mazzini 3, Desio Italy

E-mail: giul_mari@yahoo.it

Received: 18.11 .2020

Accepted: 21.01.2021

\section{Rezumat}

Este IMC un factor care contribuie la respectarea indicațiilor de chimioterapie adjuvantă în cazul cancerului rectal local avansat? $O$ analiză retrospectivă multicentrică și o comparație cu pacienți normoponderali

Context: Respectarea indicațiilor de chimioterapie adjuvantă în cazul pacienților supuşi interventiilor chirurgicale rectale variază între $43 \%$ şi 73,6\%. Motivele pentru care nu s-a început sau nu s-a finalizat chimioterapia adjuvantă cuprind apariția complicațiilor postoperatorii, toxicitatea medicamentoasă, progresia bolii şi/sau preferințele pacientului. $\mathrm{Nu}$ se cunosc multe despre impactul obezității asupra respectării indicațiilor de chimioterapie adjuvantă în acest context.

Metode: Acest studiu retrospectiv, multicentric, a analizat respectarea indicațiilor de chimioterapie adjuvantă şi morbiditatea asociată tratamentului în cazul a 511 pacienți care au suferit o intervenție chirurgicală curativă pentru cancer rectal în şase centre din Italia specializate în tratamente pentru afecțiuni colorectale, în perioada ianuarie 2013 - decembrie 2017.

Rezultate: 70 dintre pacienți erau obezi (IMC $\left.>30 \mathrm{~kg} / \mathrm{m}^{2}\right)$. Proporția procedurilor chirurgicale deschise $(22,9 \%$ vs. $13,4 \%)$ şi a 
conversiilor (14,3\% vs. $4,8 \%)$ a fost mai mare în cazul pacienților obezi, comparativ cu cei normoponderali $(p<0,001)$. Durata medie a spitalizării a fost cu o zi mai mare în cazul pacientilor obezi (9 zile vs. 10 zile, $p=0,038$ ), fără a exista vreo diferență semnificativă din punct de vedere statistic în ceea ce priveşte rata complicațiilor, atât în general (58,6\% în cazul pacienților obezi vs. 52,3\% în cazul celor normoponderali), cât şi prin prisma scorului Clavien-Dindo $\geq 3$ (17,1\% vs. 10,9\%). S-au oferit indicații de chimioterapie adjuvantă pentru 49/70 (70\%) de pacienți din grupul cu pacienți obezi şi pentru 306/441 $(69,4 \%)$ de pacienți din grupul de pacienți normoponderali $(p=0,43)$. Nu a existat o diferență semnificativă din punct de vedere statistic în ceea ce priveşte respectarea indicațiilor de chimioterapie adjuvantă: $18,4 \%$ dintre pacienții obezi, respectiv 22,9\% dintre cei normoponderali nu au început chimioterapia adjuvantă, în vreme ce $36,7 \%$, respectiv $34,6 \%$ au început-o, însă nu au finalizat tratamentul programat $(\mathrm{p}=0.79)$. Per total, $55 \%$ dintre pacienții care au început chimioterapia adjuvantă au finalizat cu succes tratamentul.

Concluzii: Obezitatea nu a influențat respectarea indicațiilor de chimioterapie adjuvantă în cazul cancerului rectal local avansat: gradul de respectare a indicatiilor a fost redus atât în cazul pacienților obezi, cât şi în cel al pacienților normoponderali, fără să existe vreo diferență majoră din punct de vedere statistic între cele două grupuri. Rata complicațiilor majore nu a fost influențată semnificativ din punct de vedere statistic de nivelul ridicat al IMC.

Cuvinte cheie: cancer rectal, obezitate, chimioterapie adjuvantă

\section{Abstract}

Background: Compliance to adjuvant chemotherapy (AC) for patients undergoing rectal surgery ranges from $43 \%$ to $73.6 \%$. Reasons reported for not initiating or completing AC include onset of postoperative complications, drug toxicity, disease progression and/or patient preferences. Little is known regarding the impact of obesity on the compliance to $\mathrm{AC}$ in this setting.

Methods: This multicenter, retrospective study analyzed compliance to AC and treatment-related morbidity in 511 patients having undergone surgery with curative intent for rectal cancer in six Italian colorectal centers between January 2013 and December 2017.

Results: 70 patients were obese $(\mathrm{BMI}>30 \mathrm{~kg} / \mathrm{m} 2)$. The proportion of open procedures $(22.9 \% \mathrm{vs}$. $13.4 \%)$ and conversions (14.3\% vs. $4.8 \%$ ) was greater in obese compared to non-obese patients $(\mathrm{p}<0.001)$. Median hospital stay was one day longer for obese patients (9 days vs. 10 days, $\mathrm{p}=0.038)$ while there was no statistically significant difference in the complication rate, whether overall (58.6\% in obese vs. $52.3 \%$ in non-obese) or with a Clavien-Dindo score $\geq 3(17.1 \%$ vs $10.9 \%)$. AC was offered to $49 / 70(70 \%)$ patients in the obese group and 306/441 (69.4\%) in the non-obese group $(\mathrm{p}=0.43)$. There was no statistically significant difference in AC compliance: $18.4 \%$ and $22.9 \%$ did not start AC, while $36.7 \%$ and $34.6 \%$, started AC but did not complete the scheduled treatment $(\mathrm{p}=0.79)$ in the obese and non-obese group, respectively. Overall, $55 \%$ of patients who started AC successfully completed their adjuvant treatment.

Conclusions: Obesity did not impact compliance to $\mathrm{AC}$ for locally advanced rectal cancer: compliance was poor in obese and non-obese patients with no statistically significant difference between the two groups. Major complication rate was not statistically significantly affected by increased BMI.

Key words: rectal cancer, obesity, adjuvant chemotherapy

\section{Introduction}

Adherence to adjuvant chemotherapy (AC) schedules for patients who undergo rectal surgery for cancer have been reported to range from $43 \%$ to $73.6 \%(1,2)$. The main reported 
reasons for not initiating or completing adjuvant therapy included the onset of postoperative complications, drug intolerance, disease progression, and patient preference (3).

The prevalence of obesity (as determined by body mass index (BMI)> $\left.30 \mathrm{~kg} / \mathrm{m}^{2}\right)$ continues to increase worldwide and in Europe (4). There is increasing data that obesity could be associated with increased risk for various cancers including rectal cancer, especially in male patients $(4,5)$.

Several studies have reported an association between obesity and oncologic outcomes in rectal cancer after AC, but few have studied the relationship between obesity and compliance to $\mathrm{AC}$ for locally advanced rectal cancer (6-9).

The main aim of this retrospective multicenter study was to evaluate the impact of obesity on the compliance to $\mathrm{AC}$ in a cohort of locally advanced rectal cancer patients undergoing surgical intervention with curative intent. The secondary goals of this study were to analyze the peri-operative complication rates and the reasons for interruption of AC.

\section{Methods}

\section{Study population and outcomes}

This retrospective cohort study included all consecutive patients who underwent surgery with curative intent for rectal cancer in six Italian colorectal centers between January 2013 and December 2017 (5 years). Patients with stage IV disease, younger than 18 years or undergoing palliative or emergency surgery were excluded from the analysis. Prospectively collected data were entered into a database specifically designed to investigate the compliance to AC. Recorded parameters included patient characteristics, patient frailty as per the Charlson comorbidity index (CCI) (10) and Robinson Score (11), pre-operative tumor stage, neo-adjuvant chemo-radiation therapy, surgical, intra-operative and postoperative parameters, early and delayed surgical complications and administration of AC. We specifically concentrated on the reasons why patients did not start, discontinued or had a modification in their $\mathrm{AC}$ regimen.
Failure was considered when AC was not started or was discontinued, when the dose was reduced, or another regimen was started due to intolerance. Obesity was defined as a $\mathrm{BMI}>30 \mathrm{~kg} / \mathrm{m}^{2}$. Data were analyzed according to four categories: underweight or normal weight (BMI $<25.0 \mathrm{~kg} / \mathrm{m}^{2}$ ), over weight (BMI $25.0-29.9 \mathrm{~kg} / \mathrm{m}^{2}$ ), obesity class I (BMI $30.0-34.9 \mathrm{~kg} / \mathrm{m}^{2}$ ), obese classes II (BMI $30.0-$ $\left.35.0 \mathrm{~kg} / \mathrm{m}^{2}\right)$ III $\left(\right.$ BMI $\left.35.0-40.0 \mathrm{~kg} / \mathrm{m}^{2}\right)$. This study was approved by the local ethical committee of each hospital.

The decision to propose neo-adjuvant and/or adjuvant CT was reached during a multidisciplinary assessment for each patient according to the National Comprehensive Cancer Network ${ }^{\circledR}$ guidelines (12). Surgery was performed 6-8 weeks after completion of neoadjuvant radiation therapy. All patients underwent either total (TME) or partial mesorectal excision depending on the location of the primary tumor. The surgical approach (open versus laparoscopic) was chosen according to surgeons' preference and expertise. A loop ileostomy was planned before surgery based on the distance of the anastomosis from the anal verge, the use of neo-adjuvant chemoradiation therapy (nCRT) and general health of the patient. Postoperative morbidity was rated according to the Clavien-Dindo (CD) classification (13). Major complications were defined as complications with a CD grade equal to or greater than 3 . Three to four weeks after surgery, patientsreceived AC (FOLFOX or CapeOX) for 6 months.

\section{Statistical Analysis}

Continuous data were reported as median and interquartile ranges (IQR) and categorical data as numbers and percentages (\%).

The chi-square test (or Fisher's exact test when appropriate) and Wilcoxon test were used, to compare categorical and continuous variables between groups, respectively.

Univariate and multivariate log-binomial regression models were performed to evaluate whether obesity status and other selected risk factors were associated with 
the risk of major complications (CD score $\geq 3$ ).

A $p$ value $<0.05$ was considered statistically significant. All analyses were performed with SAS software, version 9.4 (SAS Institute, Cary, NC).

\section{Results}

Of 511 patients corresponding to the inclusion criteria, 441 patients had a BMI less than 30 $\mathrm{kg} / \mathrm{m}^{2}$, while 70 patients were obese (BMI>30 $\mathrm{kg} / \mathrm{m}^{2}$ ). Patient and tumor related characteristics as well as operative details are reported in Table 1 . There were no statistically significant

Table 1. Patients characteristics $(\mathrm{N}=511)$

\begin{tabular}{|c|c|c|c|c|}
\hline & & Not obese PTs $(n=441)$ & Obese PTs $(n=70)$ & $p^{1}$ \\
\hline Age, median (IQR) & & $71(62,78)$ & $69(60,75)$ & 0.21 \\
\hline Charlson score, median (IQR) & & $5(4,6)$ & $5(3,7)$ & 0.98 \\
\hline \multirow[t]{2}{*}{ Number of lymphnodes, median (IQR) } & & $17(13,24)$ & $18(14,26)$ & 0.13 \\
\hline & Missing & 3 & 0 & \\
\hline $\begin{array}{l}\text { Number of positive lymphnodes (in } \mathrm{N}+\text { ), } \\
\text { median (IQR) }\end{array}$ & & $3(1,4)$ & $1(1,3)$ & 0.10 \\
\hline $\begin{array}{l}\text { Number of positive mesorectum } \\
\text { lymphnodes (in } N+\text { ), median (IQR) }\end{array}$ & & $2(1,4)$ & $1(1,2)$ & 0.21 \\
\hline BMI classes, $\mathrm{n}(\%)$ & $\begin{array}{l}\text { Underweight }\left(<18.5 \mathrm{~kg} / \mathrm{m}^{2}\right) \\
\text { Normal weight }\left(18.5-<25 \mathrm{~kg} / \mathrm{m}^{2}\right) \\
\text { Overweight }\left(25-<30 \mathrm{~kg} / \mathrm{m}^{2}\right) \\
\text { Obesity class I }\left(30-<35 \mathrm{~kg} / \mathrm{m}^{2}\right) \\
\text { Obesity class II }\left(35-<40 \mathrm{~kg} / \mathrm{m}^{2}\right) \\
\text { Obesity class III }\left(\geq 40 \mathrm{~kg} / \mathrm{m}^{2}\right)\end{array}$ & $\begin{array}{l}10(2.3) \\
236(53.5) \\
195(44.2) \\
- \\
- \\
-\end{array}$ & $\begin{array}{l}- \\
- \\
- \\
57(81.4) \\
10(14.3) \\
3(4.3)\end{array}$ & - \\
\hline Sex, N (\%) & $\begin{array}{l}\text { Female } \\
\text { Male }\end{array}$ & $\begin{array}{l}178(40.4) \\
263(59.6)\end{array}$ & $\begin{array}{l}26(37.1) \\
44(62.9)\end{array}$ & 0.70 \\
\hline Tumor site, $\mathrm{n}(\%)$ & $\begin{array}{l}\text { Low } \\
\text { Middle } \\
\text { High } \\
\end{array}$ & $\begin{array}{l}185(42.0) \\
177(40.1) \\
79(17.9) \\
\end{array}$ & $\begin{array}{l}26(37.1) \\
24(34.3) \\
20(28.6)\end{array}$ & 0.11 \\
\hline Stage, n (\%) & $\begin{array}{l}0 \\
\text { Complete response } \\
\text { I } \\
\text { IIA } \\
\text { IIB } \\
\text { IIC } \\
\text { IIIA } \\
\text { IIIB } \\
\text { IIIC }\end{array}$ & $\begin{array}{l}3(0.7) \\
28(6.3) \\
141(32.0) \\
88(20.0) \\
8(1.8) \\
0(0.0) \\
41(9.3) \\
112(25.4) \\
20(4.5)\end{array}$ & $\begin{array}{l}1(1.4) \\
3(4.3) \\
20(28.6) \\
18(25.7) \\
0(0.0) \\
1(1.4) \\
6(8.6) \\
17(24.3) \\
4(5.7)\end{array}$ & 0.27 \\
\hline Neo-adjuvant CT, n (\%) & $\begin{array}{l}\text { No } \\
\text { Yes } \\
\text { Missing }\end{array}$ & $\begin{array}{l}239(54.8) \\
197(45.2) \\
5\end{array}$ & $\begin{array}{l}38(55.9) \\
30(44.1) \\
2\end{array}$ & 0.97 \\
\hline Tumor type, n (\%) & $\begin{array}{l}\text { Adenocarcinoma } \\
\text { Mucinous }\end{array}$ & $\begin{array}{l}420(95.2) \\
21(4.8) \\
\end{array}$ & $\begin{array}{l}67(95.7) \\
3(4.3) \\
\end{array}$ & 1.00 \\
\hline \multicolumn{5}{|l|}{ Not sure the next two are needed } \\
\hline Tumor grade, n (\%) ref/ & $\begin{array}{l}\text { G1 } \\
\text { G2 } \\
\text { G3 } \\
\text { Missing }\end{array}$ & $\begin{array}{l}44(11.4) \\
278(72.2) \\
63(16.4) \\
56\end{array}$ & $\begin{array}{l}7(12.3) \\
36(63.2) \\
14(24.6) \\
13\end{array}$ & 0.28 \\
\hline Mismatch repair, n (\%) & $\begin{array}{l}\text { Absent } \\
\text { Present } \\
\text { Missing }\end{array}$ & $\begin{array}{l}44(19.7) \\
179(80.3) \\
218 \\
\end{array}$ & $\begin{array}{l}4(13.3) \\
26(86.7) \\
40\end{array}$ & 0.55 \\
\hline Type of surgery, n (\%) & $\begin{array}{l}\text { Open } \\
\text { Laparoscopic } \\
\text { Conversion }\end{array}$ & $\begin{array}{l}59(13.4) \\
361(81.9) \\
21(4.8) \\
\end{array}$ & $\begin{array}{l}16(22.9) \\
44(62.9) \\
10(14.3)\end{array}$ & $<0001$ \\
\hline Free distal margin, $\mathrm{n}(\%)$ & $\begin{array}{l}\text { No } \\
\text { Yes }\end{array}$ & $\begin{array}{l}8(1.8) \\
433(98.2)\end{array}$ & $\begin{array}{l}1(1.4) \\
69(98.6)\end{array}$ & 1.00 \\
\hline Free circumference margin, $\mathrm{n}(\%)$ & $\begin{array}{l}\text { No } \\
\text { Yes }\end{array}$ & $\begin{array}{l}31(7.0) \\
410(93.0)\end{array}$ & $\begin{array}{l}4(5.7) \\
66(94.3)\end{array}$ & 0.88 \\
\hline lleostomy, n (\%) & $\begin{array}{l}\text { No } \\
\text { Yes }\end{array}$ & $\begin{array}{l}183(41.5) \\
258(58.5)\end{array}$ & $\begin{array}{l}36(51.4) \\
34(48.6)\end{array}$ & 0.15 \\
\hline
\end{tabular}

PT: patient; CT:chemotherapy; ${ }^{1}$ Chi-square $\mathrm{p}$-value for categorical variables, Wilcoxon $\mathrm{p}$-value for continuous variables 
differences between the two groups in CCI, Robinson Score, sex, tumor site, tumor grade and stage (Table 2). Retrieved lymph nodes rates as well as the oncological quality of the pathological specimens were adequate in both groups. The proportion of open procedures and conversions was greater in obese $(22.9 \%$ and $14.3 \%)$ compared to non-obese patients (13.4\% and $4.8 \%, \mathrm{p}<0.001)$. Median hospitalization was one day longer for obese patients (9 days vs. 10 days, $p=0.038$ ) while there were no statistically significant differences in the complication rates, whether overall $(58.6 \%$ in obese vs. $52.3 \%$ in non-obese) or with a Clavien-Dindo score $\geq 3(17.1 \%$ vs. $10.9 \%)$ as reported in Table 2.

AC was offered to $49 / 70(70 \%)$ of the obese patients and to $306 / 441(69.4 \%)$ of non-obese patients $(\mathrm{p}=0.43)$. Reasons for not starting or not completing AC are described in Table 3. There was no statistically significant difference in AC compliance: $18.4 \%$ and $22.9 \%$ did not start, while $36.7 \%$ and $34.6 \%$, started but did not complete the scheduled treatment $(\mathrm{p}=0.79)$ in the obese and non-obese groups, respectively. Overall, $55 \%$ of patients who started AC successfully completed their adjuvant treatment. These results did not change even after stratification of BMI subgroups (Table 4).

From log-binomial regression analysis, nCRT, male sex and CCI emerged as risk factors for the onset of major post-operative complications for obese patients (Table 5).

There was no statistically significant difference in the reasons affecting compliance to $\mathrm{AC}$ between the two groups: $18 \%(9 / 49)$ obese vs.22\% (70/306) of non-obese patients did not start AC. Stoma related symptoms such as dehydration did not differ statistically significantly between the two groups: $12.8 \%$ in the obese vs. $14.6 \%$ in the non-obese $(p=1.00)$.

\section{Discussion}

The results of our study suggest that obesity does not affect compliance to $\mathrm{AC}$ in patients undergoing surgery with curative intent for non-metastatic rectal cancer. No statistically significant difference was found in the complication rate between obese and non-obese patients.

The association between obesity and oncological outcomes has been investigated in other types of cancer, and was found to be associated with higher rates of local recurrence, distant metastasis, and decreased overall survival $(14,15)$. Conversely, other studies described a better survival in obese patients compare to normal weight subjects, possibly due to increased tolerance to peri-operative chemoradiation (16). Weight loss during cancerrelated treatment may in fact be better tolerated by overweight subjects, avoiding the typical comorbidities derived from weakened

Table 2. Association betweenobesitystatus of patients and outcomes of interest $(\mathrm{N}=511)$

\begin{tabular}{|c|c|c|c|c|}
\hline & & Not obese PTs $(n=441)$ & Obese PTs $(n=70)$ & $\mathbf{p}^{1}$ \\
\hline \multicolumn{2}{|c|}{ Days of hospitalization, median (IQR) } & $9(6,13)$ & $10(7,16)$ & 0.038 \\
\hline \multirow[t]{8}{*}{ Clavien-Dindo, N (\%) } & 0 & $208(47.2)$ & $29(41.4)$ & - \\
\hline & 1 & $110(24.9)$ & $14(20.0)$ & \\
\hline & 2 & $75(17.0)$ & $15(21.4)$ & \\
\hline & $3 a$ & $10(2.3)$ & $4(5.7)$ & \\
\hline & $3 b$ & $28(6.3)$ & $5(7.1)$ & \\
\hline & $4 a$ & $1(0.2)$ & $1(1.4)$ & \\
\hline & $4 b$ & $0(0.0)$ & $2(2.9)$ & \\
\hline & 5 & $9(2.0)$ & $0(0.0)$ & \\
\hline \multirow[t]{3}{*}{ Relevant complications, N (\%) } & No & $208(47.2)$ & $29(41.4)$ & 0.29 \\
\hline & Minor & $185(42.0)$ & $29(41.4)$ & \\
\hline & Major & $48(10.9)$ & $12(17.1)$ & \\
\hline \multirow[t]{4}{*}{ CT compliance, $\mathrm{N}(\%)$} & Not indicated & $135(30.6)$ & $21(30.0)$ & 0.92 \\
\hline & Indicated, but not started & $70(15.9)$ & $9(12.9)$ & \\
\hline & Started, but not completed & $106(24.0)$ & $18(25.7)$ & \\
\hline & Completed & $130(29.5)$ & $22(31.4)$ & \\
\hline
\end{tabular}

PT: patient; CT: chemotherapy; ${ }^{1}$ Chi-square $p$-value for categorical variables, Wilcoxon $p$-value for continuous variables 
Table 3. Reasons for not starting or not completing adjuvant chemo-therapy

\begin{tabular}{|c|c|c|c|}
\hline & Not obese PTs $(\mathrm{N}=306)$ & Obese PTs $(\mathrm{N}=49)$ & $\mathbf{P}^{1}$ \\
\hline $\begin{array}{c}\text { AC started, } \mathrm{N}(\%) \\
\text { No } \\
\text { Yes }\end{array}$ & $\begin{array}{c}70(22.9) \\
236(77.12)\end{array}$ & $\begin{array}{c}9(18.4) \\
40(81.6)\end{array}$ & 0.58 \\
\hline $\begin{array}{l}\text { Reasons for not starting } \mathrm{AC}, \mathrm{N}(\%)^{2} \\
\text { Age } \\
\text { Complications } \\
\text { General conditions } \\
\text { Patient choice }\end{array}$ & $\begin{array}{c}48(15.7) \\
10(3.3) \\
52(17.0) \\
6(2.0)\end{array}$ & $\begin{array}{l}6(12.2) \\
4(8.2) \\
6(12.2) \\
0(0.0)\end{array}$ & $\begin{array}{l}0.67 \\
0.11 \\
0.53 \\
1.00\end{array}$ \\
\hline $\begin{array}{c}\text { AC completed, } \mathrm{N}(\%)^{3} \\
\text { No } \\
\text { Yes }\end{array}$ & $\begin{array}{l}106(44.9) \\
130(55.1)\end{array}$ & $\begin{array}{l}18(45.0) \\
22(55.0)\end{array}$ & 1.00 \\
\hline $\begin{array}{l}\text { Changes in } \mathrm{AC} \text { regimen, } \mathrm{N}(\%)^{2} \\
\text { Shortened } \\
\text { Dose lowered } \\
\text { Change of } \mathrm{AC} \text { agent }\end{array}$ & $\begin{array}{l}55(23.3) \\
62(26.3) \\
30(12.7)\end{array}$ & $\begin{array}{l}6(15.0) \\
9(22.5) \\
6(15.0)\end{array}$ & $\begin{array}{l}0.31 \\
0.70 \\
0.62\end{array}$ \\
\hline $\begin{array}{l}\text { Other events } \mathrm{N}(\%)^{2} \\
\text { Readmission } \\
\text { AC where surgery performed } \\
\text { Ileostomy closed before AC } \\
\text { Dehydratation } \\
\text { Ileus } \\
\text { Mechanical occlusion } \\
\text { Ileostomy prolapse } \\
\text { Incisional hernia } \\
\text { Late anastomotic leak } \\
\text { Infection } \\
\text { AC toxicity }\end{array}$ & $\begin{array}{c}33(14.2) \\
179(76.5) \\
23(10.2) \\
34(14.6) \\
9(3.9) \\
5(2.1) \\
8(3.4) \\
15(6.4) \\
4(1.7) \\
32(13.7) \\
101(43.3)\end{array}$ & $\begin{array}{c}2(5.1) \\
31(77.5) \\
3(7.9) \\
5(12.8) \\
0(0.0) \\
0(0.0) \\
0(0.0) \\
3(7.7) \\
0(0.0) \\
1(2.6) \\
16(41.0)\end{array}$ & $\begin{array}{l}0.19 \\
1.00 \\
1.00 \\
1.00 \\
0.37 \\
1.00 \\
0.61 \\
0.73 \\
1.00 \\
0.06 \\
0.86\end{array}$ \\
\hline
\end{tabular}

PT: patient; AC: adjuvant chemotherapy; ${ }^{1}$ Fisher exact p-value; ${ }^{2}$ Multiple answers are possible; ${ }^{3}$ Based on number of PTs who started AC

Table 4. Association between obesity status of patients and outcomes of interest $(\mathrm{N}=511)$

\begin{tabular}{|c|c|c|c|c|c|c|}
\hline & & $\begin{array}{c}\text { Underweight or normal } \\
\text { weight PTs }(\mathrm{N}=246)\end{array}$ & $\begin{array}{l}\text { Over weight PTs } \\
(\mathrm{N}=195)\end{array}$ & $\begin{array}{l}\text { Obese I PTs } \\
\quad(N=57)\end{array}$ & $\begin{array}{c}\text { Obese II/III PTs } \\
(\mathrm{N}=13)\end{array}$ & $\mathbf{P}^{1}$ \\
\hline Days of hospitalization, median (IQR) & Missing & $\begin{array}{c}9(7,14) \\
1\end{array}$ & $\begin{array}{c}8(5,13) \\
1\end{array}$ & $\begin{array}{c}10(8,16) \\
0\end{array}$ & $\begin{array}{c}8(7,16) \\
0\end{array}$ & 0.41 \\
\hline Clavien-Dindo, N (\%) & $\begin{array}{l}0 \\
1 \\
2 \\
3 a \\
3 b \\
4 a \\
4 b \\
5\end{array}$ & $\begin{array}{c}123(50.0) \\
57(23.2) \\
40(16.3) \\
4(1.6) \\
16(6.5) \\
0(0.0) \\
0(0.0) \\
6(2.4)\end{array}$ & $\begin{array}{c}85(43.6) \\
53(27.2) \\
35(17.9) \\
6(3.1) \\
12(6.2) \\
1(0.5) \\
0(0.0) \\
3(1.5)\end{array}$ & $\begin{array}{c}24(42.1) \\
9(15.8) \\
14(24.6) \\
4(7.0) \\
5(8.8) \\
1(1.8) \\
0(0.0) \\
0(0.0)\end{array}$ & $\begin{array}{l}5(38.5) \\
5(38.5) \\
1(7.7) \\
0(0.0) \\
0(0.0) \\
0(0.0) \\
2(15.4) \\
0(0.0)\end{array}$ & - \\
\hline Grade of complications, N (\%) & $\begin{array}{l}\text { No } \\
\text { Minor }(<3) \\
\text { Major }(>2)\end{array}$ & $\begin{array}{l}123(50.0) \\
97(39.4) \\
26(10.6)\end{array}$ & $\begin{array}{l}85(43.6) \\
88(45.1) \\
22(11.3)\end{array}$ & $\begin{array}{l}24(42.1) \\
23(40.4) \\
10(17.5)\end{array}$ & $\begin{array}{l}5(38.5) \\
6(46.2) \\
2(15.4)\end{array}$ & 0.22 \\
\hline CT compliance, N (\%) & $\begin{array}{l}\text { Not indicated } \\
\text { Indicated, but not started } \\
\text { Started, but not completed } \\
\text { Completed }\end{array}$ & $\begin{array}{l}71(28.9) \\
46(18.7) \\
61(24.8) \\
68(27.6) \\
\end{array}$ & $\begin{array}{l}64(32.8) \\
24(12.3) \\
45(23.1) \\
62(31.8)\end{array}$ & $\begin{array}{c}15(26.3) \\
8(14.0) \\
15(26.3) \\
19(33.3) \\
\end{array}$ & $\begin{array}{l}6(46.2) \\
1(7.7) \\
3(23.1) \\
3(23.1) \\
\end{array}$ & 0.30 \\
\hline
\end{tabular}

PT: patient; CT: chemo-therapy; ${ }^{1} \mathrm{P}$-value from logistic regression for ordinal response

immune systems (17). With regard to rectal cancer, only few studies $(5,6)$ reported an association between obesity and oncologic outcomes in rectal cancer, and even fewer inquired the relationship between obesity and compliance to $\mathrm{AC}$ for locally advanced rectal cancer (18).
Compliance to $\mathrm{AC}$ is known to be affected by the rate of peri-operative complications and conversion to open surgery rate since they could translate into a possible delay in adjuvant chemotherapy onset and therefore a possible reduction of completion rate.

Of note in our study is the alarming low 
Table 5. Association between patient characteristics and onset of major (Clavien-Dindo score $>2$ ) complications according to log-binomial univariable regression analysis $(\mathrm{N}=511)$

\begin{tabular}{|c|c|c|c|c|c|c|}
\hline Variable & & $\begin{array}{c}\text { n. of relevant } \\
\text { complications / N tot }\end{array}$ & $\%$ & RR & $95 \% \mathrm{Cl}$ & $\mathbf{P}$ \\
\hline All PTs & & $60 / 511$ & 12 & & & \\
\hline Age (years) & +10 years & & & 1.12 & $0.91-1.39$ & 0.29 \\
\hline Charlson score & +2 points & & & 1.30 & $1.07-1.58$ & 0.009 \\
\hline Obesity status $^{1}$ & $\begin{array}{l}\text { No obese PTs } \\
\text { Obese PTs }\end{array}$ & $\begin{array}{c}48 / 441 \\
12 / 70\end{array}$ & $\begin{array}{l}11 \\
17\end{array}$ & $\begin{array}{l}1.00 \\
1.57\end{array}$ & $0.88-2.81$ & 0.12 \\
\hline Obesity status $^{2}$ & $\begin{array}{l}\text { Underweight or normal weight } \\
\text { Over weight } \\
\text { Obesity class I } \\
\text { Obesity class II/III }\end{array}$ & $\begin{array}{c}26 / 246 \\
22 / 195 \\
10 / 57 \\
2 / 13\end{array}$ & $\begin{array}{l}11 \\
11 \\
18 \\
15\end{array}$ & $\begin{array}{l}1.00 \\
1.07 \\
1.66 \\
1.46\end{array}$ & $\begin{array}{l}0.62-1.82 \\
0.85-3.24 \\
0.39-5.48\end{array}$ & $\begin{array}{l}0.81 \\
0.14 \\
0.58\end{array}$ \\
\hline$\overline{\operatorname{Sex}}$ & $\begin{array}{l}\text { Female } \\
\text { Male }\end{array}$ & $\begin{array}{l}15 / 204 \\
45 / 307\end{array}$ & $\begin{array}{c}7 \\
15 \\
\end{array}$ & $\begin{array}{l}1.00 \\
1.99 \\
\end{array}$ & $1.14-3.48$ & 0.015 \\
\hline Tumor site & $\begin{array}{l}\text { Low } \\
\text { Middle } \\
\text { High }\end{array}$ & $\begin{array}{c}27 / 211 \\
25 / 201 \\
8 / 99\end{array}$ & $\begin{array}{c}13 \\
12 \\
8\end{array}$ & $\begin{array}{l}1.00 \\
0.97 \\
0.63\end{array}$ & $\begin{array}{l}0.58-1.62 \\
0.30-1.34\end{array}$ & $\begin{array}{l}0.91 \\
0.23\end{array}$ \\
\hline Tumor stage & $\begin{array}{l}0 \\
1 \\
I I \\
\text { III }\end{array}$ & $\begin{array}{c}6 / 35 \\
18 / 161 \\
14 / 115 \\
22 / 200\end{array}$ & $\begin{array}{l}17 \\
11 \\
12 \\
11\end{array}$ & $\begin{array}{l}1.00 \\
0.65 \\
0.71 \\
0.64\end{array}$ & $\begin{array}{l}0.28-1.52 \\
0.30-1.71 \\
0.28-1.47\end{array}$ & $\begin{array}{l}0.32 \\
0.44 \\
0.29\end{array}$ \\
\hline Neo-adjuvant CT & $\begin{array}{l}\text { No } \\
\text { Yes } \\
\text { Missing }\end{array}$ & $\begin{array}{c}23 / 277 \\
37 / 227 \\
0 / 4\end{array}$ & $\begin{array}{c}8 \\
16 \\
0\end{array}$ & $\begin{array}{c}1.00 \\
1.96 \\
-\end{array}$ & $1.20-3.20$ & 0.007 \\
\hline Tumor type & $\begin{array}{l}\text { Adenocarcinoma } \\
\text { Mucinous }\end{array}$ & $\begin{array}{c}56 / 487 \\
4 / 24\end{array}$ & $\begin{array}{l}11 \\
17\end{array}$ & $\begin{array}{l}1.00 \\
1.45\end{array}$ & $0.57-3.67$ & 0.43 \\
\hline Tumor grade & $\begin{array}{l}\text { G1 } \\
\text { G2 } \\
\text { G3 } \\
\text { Missing }\end{array}$ & $\begin{array}{c}7 / 51 \\
25 / 314 \\
15 / 77 \\
13 / 69\end{array}$ & $\begin{array}{c}14 \\
8 \\
19 \\
19\end{array}$ & $\begin{array}{c}1.00 \\
0.58 \\
1.42 \\
-\end{array}$ & $\begin{array}{l}0.26-1.27 \\
0.62-3.24\end{array}$ & $\begin{array}{l}0.17 \\
0.41\end{array}$ \\
\hline Mismatch repair & $\begin{array}{l}\text { Absent } \\
\text { Present } \\
\text { Missing }\end{array}$ & $\begin{array}{c}5 / 48 \\
16 / 205 \\
39 / 258\end{array}$ & $\begin{array}{c}10 \\
8 \\
15 \\
\end{array}$ & $\begin{array}{c}1.00 \\
0.75 \\
-\end{array}$ & $0.29-1.94$ & 0.55 \\
\hline Type of surgery & $\begin{array}{l}\text { Open } \\
\text { Laparoscopic } \\
\text { Conversion }\end{array}$ & $\begin{array}{c}14 / 75 \\
39 / 405 \\
7 / 31\end{array}$ & $\begin{array}{l}19 \\
10 \\
23\end{array}$ & $\begin{array}{l}1.00 \\
0.52 \\
1.21\end{array}$ & $\begin{array}{l}0.30-0.90 \\
0.54-2.71\end{array}$ & $\begin{array}{l}0.02 \\
0.64\end{array}$ \\
\hline Free distal margin & $\begin{array}{l}\text { No } \\
\text { Yes }\end{array}$ & $\begin{array}{c}2 / 9 \\
58 / 502\end{array}$ & $\begin{array}{l}22 \\
12\end{array}$ & $\begin{array}{l}1.00 \\
0.52\end{array}$ & $0.15-1.81$ & 0.30 \\
\hline Free circumference margin & $\begin{array}{l}\text { No } \\
\text { Yes }\end{array}$ & $\begin{array}{c}7 / 35 \\
53 / 476\end{array}$ & $\begin{array}{l}20 \\
11\end{array}$ & $\begin{array}{l}1.00 \\
0.56\end{array}$ & $0.27-1.13$ & 0.11 \\
\hline lleostomy & $\begin{array}{l}\text { No } \\
\text { Yes }\end{array}$ & $\begin{array}{l}24 / 219 \\
36 / 292\end{array}$ & $\begin{array}{l}11 \\
12\end{array}$ & $\begin{array}{l}1.00 \\
1.12\end{array}$ & $0.69-1.83$ & 0.63 \\
\hline
\end{tabular}

RR: relative risk; Cl: confidence interval; PT: patient; CT: chemotherapy; ${ }^{1,2}$ Obese status into two categories: $\mathrm{AlC}=371.63, \log \mathrm{L}=-183.81$; obese status into four categories: $\mathrm{AIC}=375.54, \log \mathrm{L}=-183.77$. p-value LRT $(2 \mathrm{gdl})=0.96$

rate of full compliance to adjuvant treatment in patients operated for locally advanced rectal cancer, whether the patients were obese or not, confirming the data already reported in the literature regarding inadequate adherence to AC (21,22). Notwithstanding, our overall $55 \%$ adherence rate compares favorably with the literature. Fourty-three\% of patients who were randomized to receive postoperative chemotherapy finally received the planned dose within the scheduled time interval. More than a quarter of patients could not even start adjuvant chemotherapy due to postoperative complications, absence of tumour resection, disease progression, or patient refusal). Unsatisfactory compliance to $\mathrm{AC}$ does not seem to be related to the type of patients receiving the treatment (23). The role of neoadjuvant therapy (24) should be taken into consideration to improve compliance in patients with locally advance rectal cancer.

The main causes of incomplete AC did not differ significantly between the two groups. The data we found are quite in line with those reported in the literature $(21,22)$. Patient age is no longer considered as an absolute 
contraindication to adjuvant chemotherapy (25), while post-operative complications and general conditions have excluded a considerable number of patients from adjuvant therapy.

Chemotherapy toxicity and patient dehydration (mainly due to increased ostomy output) were found to be the main factors of deteriorated compliance to AC, similar to what was observed in the EORCT (21) and Chronicle (22) trials, but the BMI of patients in these two randomized studies was not detailed.

We found that obese patients treated with nCRT were at higher risk for the onset of postoperative complications (overall and major complications (Clavien-Dindo $\geq 3$ ). This result could be explained by the technical difficulty of operating obese patients with irradiated pelvis compared to non-obese patients. Interestingly, this increase in the complication rate did not translate into a reduced compliance to AC.

The poor compliance to $\mathrm{AC}$ in our reported population undergoing surgery for rectal cancer suggests that a change in treatment should be implemented moving towards a totally neo-adjuvant therapy setting.

Our study has several limitations. Data were reviewed and analyzed retrospectively; the obese group is substantially smaller than the non-obese group. Moreover, we did not study oncological outcomes such as diseasefree survival and overall survival. Our aim was to study the actual compliance of obese patients to AC after rectal cancer surgery, as we believe this information is missing from the current literature, and may be one of importance for daily clinical practice, and in particular, for patient information.

\section{Conclusion}

Obesity did not impact compliance to adjuvant chemotherapy for locally advanced rectal cancer: compliance was quite poor in both obese and non-obese patients. Increased BMI was not associated with increased major complications, while pre-operative chemoradiation therapy was found to be a risk factor for developing major complications in obese patients.

\section{Conflict of Interest}

All authors declare that they have not hing to disclose.

\section{Ethics Approval}

The study was approved by the local ethics committee of the each hospitals.

\section{References}

1. Glynne-Jones R, Counsell N, Quirke P, Mortensen N, Maraveyas A, Meadows HM, et al. Chronicle: results of a randomised phase I I I trial in locally advanced rectal cancer after neoadjuvant chemoradiation randomising postoperative adjuvant capecitabine plus oxaliplatin (XELOX) versus control. Ann Oncol. 2014;25(7):13561362

2. Breugom AJ, van Gijn W, Muller EW, Berglund T, van den Broek CBM, Fokstuen T, et al. Adjuvant chemotherapy for rectal cancer patients treated with preoperative (chemo)radiotherapy and total mesorectal excision: a Dutch Colorectal Cancer Group (DCCG) randomized phase III trial. Ann Oncol. 2015;26(4):696-701. Epub 2014 Dec 5.

3. Marques A, Peralta M, Naia A, Loureiro N, de MatosMG. Prevalence of adult overweight and obesity in 20 European countries, 2014. Eur J Public Health. 2018;28(2):295-300.

4. Pischon T, Nimptish K Obesity and Risk of Cancer: An Introductory Overview. Recent Results Cancer Res 2016;208:1-15

5. Larsson SC, Wolk A. Obesity and colon and rectal cancer risk: a meta-analysis of prospective studies. Am J Clin Nutr. 2007;86(3): 556-65.

6. Dignam JJ, Polite BN, Yothers G, Raich P, Colangelo L, O'Connell MJ, Wolmark N. Body mass index and outcomes in patients who receive adjuvant chemotherapy for colon cancer. J Natl Cancer Inst. 2006;98(22):1647-54.

7. Sinicrope FA, Foster NR, Yothers G, Benson A, Seitz JF, Labianca R, et al. Adjuvant Colon Cancer Endpoints (ACCENT) Group. Body mass index at diagnosis and survival among colon cancer patients enrolled in clinical trials of adjuvant chemotherapy. Cancer. 2013; 119(8):1528-36.

8. Sun Y, Xu Z, Lin H, Lu X, Huang Y, Huang S, et al. Impact of body mass index on treatment outcome of neoadjuvant chemoradiotherapy in locally advanced rectal cancer. Eur J Surg Oncol. 2017; 43(10):1828-1834.

9. Lee CS, Murphy DJ, McMahon C, Nolan B, Cullen G, Mulcahy H, et al. Visceral Adiposity is a Risk Factor for Poor Prognosis in Colorectal Cancer Patients Receiving Adjuvant Chemotherapy. J Gastrointest Cancer. 2015;46(3):243-50.

10. Bannay $A$, Chaignot $C$, Blotière $P O$, Basson $M$, Weill $A$, Ricordeau $P$, et al. The Best Use of the Charlson Comorbidity Index With Electronic Health Care Database to Predict Mortality.Med Care. 2016;54(2):188-94.

11. Robinson TN, Wu DS, Pointer L, Dunn CL, Cleveland JC Jr, Moss M. Simple frailty score predicts post-operative complications across surgical specialties. Am J Surg. 2013:206(4):544-550.

12. National Comprehensive Cancer Network®. Rectal Cancer (Version 4.2017). 
13. Dindo D, Demartines N, Clavien P. Classification of surgical complications: a new proposal with evaluation in a cohort of 6336 patients and results of a survey. Ann Surg. 2004;240:205-213.

14. Ewertz M, Jensen MB, Gunnarsdottir KA, Hojris I, Jakobsen EH, Nielsen D, et al. Effect of obesity on prognosis after early-stage breast cancer. J Clin Oncol. 2011;29:25-31.

15. Chan DS, Vieira AR, Aune D, Bandera EV, Greenwood DC, McTiernan $A$, et al. Body mass index and survival in women with breast cancer-systematic literature review and meta-analysis of 82 follow-up studies. Ann Oncol. 2014;25:1901-1914.

16. Bergom C, Kelly T, Bedi M, Saeed H, Prior P, Rein LE, et al. Association of locoregional control with high body mass index in women undergoing breast conservation therapy for early-stage breast cancer. Int J Radiat Oncol Biol Phys. 2016;96:65-71.

17. Liu J, Deng YT, Zhang L, Li N, Jiang M, Zou LQ, et al. Body mass index as a prognostic factor in patients with extranodal natural killer/T-cell lymphoma, nasal type. Oncotarget. 2016;7:78159- 78167.

18. Meyerhardt JA, Catalano PJ, Haller DG, Mayer RJ, Benson AB, 3rd Macdonald JS, Fuchs CS. Influence of body mass index on outcomes and treatment-related toxicity in patients with colon carcinoma. Cancer. 2003;98(3):484-95.

19. Sinicrope FA, Foster NR, Sargent DJ, O'Connell MJ, Rankin C. Obesity is an independent prognostic variable in colon cancer survivors. Clin Cancer Res. 2010;16(6):1884-93.

20. Baastrup NN, Christensen JK, Jensen KK, Jørgensen LN. Visceral obesity and short-term outcomes after laparoscopic rectal cancer resection. Surg Endosc. 2020;34(1):177-185. Epub 2019 Mar 18.

21. Bosset JF, Calais G, Mineur L, Maingon P, Stojanovic-Rundic S, Bensadoun RJ, et al. Fluorouracil-based adjuvant chemotherapy after preoperative chemoradiotherapy in rectal cancer: long-term results of the EORTC 22921 randomised study. Lancet Oncol. 2014;15(2):184-88.

22. Glynne-Jones R, Counsell N, Quirke P, Mortensen N, Maraveyas A, Meadows HM, et al. Chronicle: results of a randomised phase III trial in locally advanced rectal cancer after neoadjuvant chemoradiation randomising postoperative adjuvant capecitabine plus oxaliplatin (XELOX) versus control. Ann Oncol. 2014;25(7): 1356-62.

23. Breugom AJ, van Gijn W, Muller EW, Berglund A, van den Broek CB, Fokstuen T, et al. Adjuvant chemotherapy for rectal cancer patients treated with preoperative (chemo)radiotherapy and total mesorectal excision: a Dutch Colorectal Cancer Group (DCCG) randomized phase III trial. Ann Oncol. 2015;26(4):696-701.

24. Zaborowski A, Stakelum A, Winter DC. Systematic review of outcomes after totalneoadjuvant therapy for locally advanced rectal cancer. Br J Surg. 2019;106(8):979-987.

25. Papamichael D, Audisio RA, Glimelius B, de Gramont A, Glynne-Jones $\mathrm{R}$, Haller $\mathrm{D}$, et al. Treatment of colorectal cancer in older patients: International Society of Geriatric Oncology (SIOG) consensus recommendations 2013. Ann Oncol. 2015;26(3):463-76. 